\title{
An Ingestion of Industrial Strength Hydrogen Peroxide Resulting in Cerebral Embolism and Death
}

\author{
Muhammad Sohaib Afzal ${ }^{1, *}$, Saleha Sharif Dar ${ }^{1}$, Tayyaba Sarwar ${ }^{1}$, Muhammad Zubair Afzal ${ }^{2}$ \\ ${ }^{1}$ Quaid-e-Azam Medical College, Bahawalpur, Pakistan \\ ${ }^{2}$ Dartmouth-Hitchcock Medical Center, Lebanon, NH USA \\ *Corresponding author: Muhammad.Afzal@hitchcock.org
}

\begin{abstract}
Hydrogen peroxide is a well-known remedy used in homeopathic medicine. Accidental ingestion can have a broad range of side effects depending upon the quantity and concentration of ingestion. Air emboli to the brain have been reported in very few case reports. We present an interesting case of accidental ingestion of $35 \%$ hydrogen peroxide resulted in ischemic stroke and seizures due to air embolism. Our patient was an elderly woman who accidentally drank industrial strength hydrogen peroxide thinking of water. Upon ingestion of approximately two tablespoons of hydrogen peroxide, she developed respiratory distress within few minutes. She was reported to have a frothy mouth and soon became unresponsive. EMS arrived and intubated her on her way to the hospital. A CT of the chest/abdomen/pelvis showed air in the heart, spleen, splenic and portal veins. A CT, MRI of head and EEG were performed to evaluate her altered mental status. She was treated with phenytoin, midazolam for seizure prophylaxis and hyperbaric oxygen for the air embolism without improvement. The family ultimately decided to transition to comfort measures only. This is a rare case of air embolism. About $20 \%$ of air-embolism occurs in contrast enhanced CT's. Very rarely it can be because of hydrogen peroxide poisoning. Hydrogen peroxide has many common uses but has a great potential for misuse. Hyperbaric oxygen has a role in both limiting damage and enhancing recovery. This is the current standard of care as documented in previously reported case.
\end{abstract}

Keywords: hydrogen peroxide, cerebral embolism, air embolism, ischemic stroke

Cite This Article: Muhammad Sohaib Afzal, Saleha Sharif Dar, Tayyaba Sarwar, and Muhammad Zubair Afzal, "An Ingestion of Industrial Strength Hydrogen Peroxide Resulting in Cerebral Embolism and Death." American Journal of Medical Case Reports, vol. 5, no. 9 (2017): 234-236. doi: 10.12691/ajmcr-5-9-1.

\section{Introduction}

Hydrogen peroxide, an oxidizing agent, is used in several household products, including disinfectants, chlorine-free bleaches, fabric stain removers, contact lens disinfectants and hair dyes, and some tooth whitening products as well $[1,2]$.

Medicinal uses of Hydrogen peroxide include wound irrigation and sterilization of ophthalmic and endoscopic instruments $[1,2]$. There are three primary mechanisms by which hydrogen peroxide causes toxicity, namely: corrosive damage, oxygen gas formation and lipid peroxidation [2]. While exposure results in local tissue damage by caustic action, ingestion of concentrated $(>35 \%)$ hydrogen peroxide can also lead to the generation of substantial volumes of oxygen. When the amount of oxygen evolved exceeds its maximum solubility in blood, venous or arterial gas embolism may occur [3]. We report a case in which an elderly woman presents with ischemic stroke due to air embolism after ingesting unlabeled 35\% Hydrogen Peroxide.

\section{Case Report}

This is a case of an 89-year-old very functional woman. She had a history of hypertension, hyperlipidemia, moderate aortic stenosis osteoporosis, atrial fibrillation and Meniere's disease. She was visiting her daughter's house and woke up in the middle of the night feeling thirsty. She got out of bed to get some water from the refrigerator. She accidentally ingested unlabeled industrial strength hydrogen peroxide $(35 \%)$ rather than water. Upon ingestion of approximately $30 \mathrm{ml}$ of hydrogen peroxide, she noticed a terrible taste and soon after developed respiratory distress. She began to froth at her mouth and soon after, she became unresponsive. Emergency response service was called, and she was found to be obtunded by the response team. She underwent endotracheal intubation due to the concern of airway compromise and transferred to our facility. On arrival to the emergency department, she was basic lab work was unremarkable. EKG was unremarkable as well. Her altered mental status was evaluated with a head CT and brain MRI. Cerebral air emboli can sometimes be identified as multiple low-density lesions on brain CT with multiple subsequent infarcts in the areas of emboli. Patient's brain imaging demonstrated multiple areas of cerebral ischemia [Figure 1]. A CT of the chest/abdomen/pelvis showed air in the heart, spleen, splenic vein, and portal vein.

Venous air emboli appear as hypodense bubbles of air at the most anterior aspects of hyper-dense contrast filled vessels and heart chambers as shown [Figure 2] 


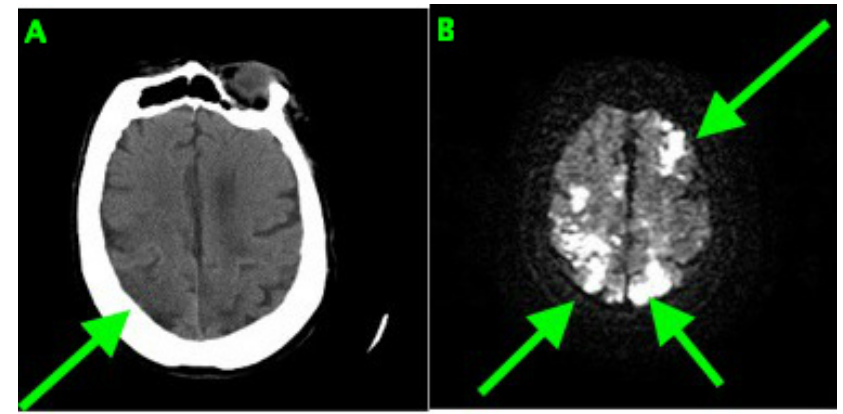

Figure 1. CT head showing area of infarct in the occipital region in 1A and MRI brain showing multiple areas with air embolism in 1B

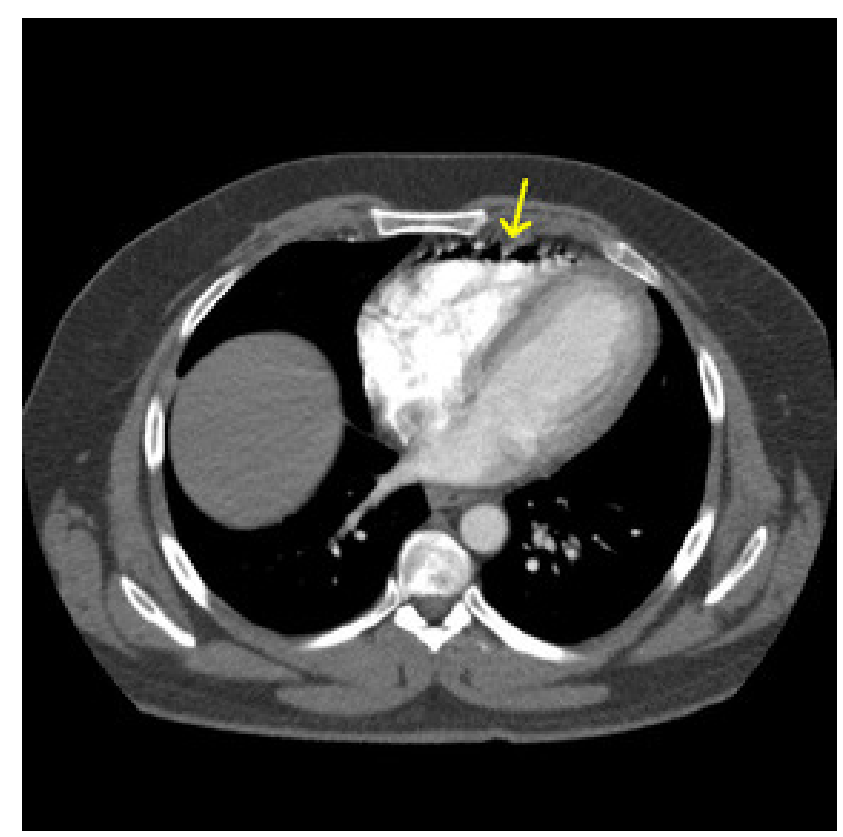

Figure 2. CT chest with air in the heart.

The patient has continued the ventilatory support. Neurology and toxicology were consulted. She was recommended hyperbaric oxygen treatment that was applied. She was also started on phenytoin and midazolam for seizure prophylaxis. Despite hyperbaric oxygen therapy, the patient could not regain consciousness and could not recover. Her prognosis remained poor. Eventually family decided to withdraw care per patient's wishes. The patient passed away peacefully within 48 hours of her presentation.

\section{Discussion}

Hydrogen peroxide, an oxidizing agent, is a colorless liquid at room temperature [1]. It is most commonly available as a solution in water and found in cleaning and bleaching products at concentrations of $3-5 \%$, but can be found in higher concentrations of $35-90 \%$ in industrial strength products $[1,2]$. Severe complications are rare with $3-5 \%$ concentrated hydrogen peroxide, but ingestion can cause respiratory tract irritation, gastrointestinal mucosal damage, vomiting, and gastric distention [2]. Children are the most frequent victims of the accidental ingestion of the household hydrogen peroxide [3]. In a study, 60\% hydrogen peroxide exposures have been reported in the children, and $85 \%$ of such exposures have resulted from the ingestion. [2]

Severe toxicities have been reported with highly concentrated hydrogen peroxide (35-90\%). Higher strength hydrogen peroxide $(35-90 \%)$ can cause complications like severe chemical burns to the gastrointestinal tract, colitis, intestinal gangrene, rupture, seizures, cerebral infarction, and in extreme cases cardiac arrest, hypotension and even death $[4,5]$.

Hydrogen peroxide is a thermodynamically unstable compound. It is rapidly decomposed through an exothermic reaction. This mechanism leading to oxygen embolus formation from hydrogen peroxide is the decomposition of $\mathrm{H}_{2} \mathrm{O}_{2}$ to water and oxygen $\left(\mathrm{H}_{2} \mathrm{O}_{2} \rightarrow 2 \mathrm{H}_{2} \mathrm{O}+\mathrm{O}_{2}\right)$. The reaction is further potentiated by tissue catalases and membrane peroxidases acting on $\mathrm{H}_{2} \mathrm{O}_{2}$ [6,7]. The amount of oxygen released from the ingestion of hydrogen peroxide will depend on the ingested quantity of the hydrogen peroxide. Watt BE et al. reported that ingestion of $30 \mathrm{~mL}$ of hydrogen peroxide would result in $3.5 \mathrm{~L}$ of oxygen production. [2] When oxygen concentration exceeds its maximum solubility in blood, it leads to gas embolism especially in the portal venous system, gastric wall, and brain as was demonstrated in our patient $[8,9]$.

In our case patient developed cerebral infarction due to cerebral air gas embolism. No other complications were observed. The patient had a short hospital course, and it is likely that no other adverse event was identified.

Gas embolism to the brain manifests similarly as any other embolic ischemic stroke [8,10].

A cerebral arterial gas embolism is hard to distinguish as a cause of ischemic stroke from other forms of embolic stroke, and the diagnosis requires a high level of suspicion [10]. Management of hydrogen peroxide ingestion depends on the severity of ingestion and systems involved. Stomach decontamination is not achievable due to rapid decomposition of hydrogen peroxide. A detailed history is critical. Accidental vs. intentional ingestion should always be kept in mind when dealing with suspected gas embolism and hydrogen peroxide poisoning should be the first one coming in mind. Routine clinical investigations like blood gas, chest radiography, CT scan, EKG, CT/MRI should be performed $[9,10]$. CT/MRI typically will show the gas emboli, but these tests may prove unproductive, especially if there is a delay in imaging [10].

Hyperbaric oxygen therapy (HBOT) has been successfully used in the treatment of cerebral air gas embolism (CAGE) due to concentrated hydrogen peroxide $[11,12]$. HBOT minimizes the neurological insult by cerebral vasoconstriction, a decrease in intracranial pressure, and reduction in the leukocytes adherence to cerebral endothelium. HBOT results in reactive $\mathrm{O}_{2}$ and nitrogen species production that promote synthesis of vascular endothelial growth factors, hypoxia inhibitory factor (HIF) related gene products resulting in neovascularization. These reactive species also facilitate activation of the neutrophil $\beta$-Actin S-nitrosylation, lower the monocyte chemokine synthesis as well as ischemic pre-conditional changes via production of HIF-1 causing a diminished inflammatory response. All these modifications improve the post-ischemic tissue survival [13]. All these factors assist in curtailing the neurological damage. 
We can conclude that ingestion of concentrated hydrogen peroxide can result in cerebral air gas embolism with clinical and radiological evidence. Early treatment with hyperbaric oxygen therapy can lessen the damage, but the ingestion of the higher quantities may be life threatening. Public awareness is essential to curb the future incidents, and it should be stressed that it should be stored in a secure place.

\section{References}

[1] "65 Brilliant Uses For Hydrogen Peroxide. Every Home Should Have A Bottle!" Juicing for Health, 28 July 2017, juicing-forhealth.com/65-uses-for-hydrogen-peroxide. Accessed 24 Aug. 2017.

[2] Watt, BE., Alex T. Proudfoot, and J. Allister Vale. "Hydrogen Peroxide Poisoning.” Toxicological Reviews 23.1. 51-57. 2004.

[3] Pritchett, Sean, Daniel Green, and Peter Rossos. "Accidental Ingestion of $35 \%$ Hydrogen Peroxide." Canadian Journal of Gastroenterology 21.10, 665-67. 2007.

[4] Sherman, S. J., L. V. Boyer, and W. A. Sibley. "Cerebral Infarction Immediately after Ingestion of Hydrogen Peroxide Solution." Stroke 25.5 (1994): 1065-067.
[5] Steven P. Rider, Sue B. Jackson and Daniel E. Rusyniak "Cerebral air gas embolism from concentrated hydrogen peroxide ingestion.” Clinical Toxicology (2008): 46:9, 815-818.

[6] Humberston, C. Lynn, Bonnie S. Dean, and Edward P. Krenzelok. "Ingestion of $35 \%$ hydrogen peroxide." Journal of Toxicology: Clinical Toxicology 28, no. 1 (1990): 95-100.

[7] “Air embolism." Air embolism, www.uptodate.com/contents/airembolism. Accessed 24 Aug. 2017.

[8] Zhang, Junjie, Chengliang Zhang, and Jianqin Yan. "Massive Cerebral Gas Embolism under Discectomy due to Hydrogen Peroxide Irrigation." Case Reports in Neurological Medicine 2015 (2015): 1-4.

[9] Ahluwalia, R.s., and I.1.h. Reichert. "Primary oxygen embolism after hydrogen peroxide irrigation of a large wound.” Injury Extra, vol. 37, no. 4, pp. 139-140. 2006.

[10] Jeon, S.-B., and D.-W. Kang. "Cerebral air emboli on T2-weighted gradient-echo magnetic resonance imaging." Journal of Neurology, Neurosurgery \& Psychiatry 78, no. 8 (2007): 871.

[11] Jain, K. K. "Cerebral Air Embolism." Textbook of Hyperbaric Medicine. 121-30. 2016

[12] Mullins, Michael E., and Jeanelle T. Beltran. "Acute Cerebral Gas Embolism from Hydrogen Peroxide Ingestion Successfully Treated with Hyperbaric Oxygen." Journal of Toxicology: Clinical Toxicology 36, no. 3 (1998): 253-56.

[13] Thom, Stephen R. "Hyperbaric Oxygen: Its Mechanisms and Efficacy." Plastic and Reconstructive Surgery, vol. 127, 2011. 